\title{
DYNAMICAL STABILITY AND DYNAMICAL EVOLUTION OF THE DISKS OF SC GALAXIES
}

\author{
B. FUCHS, R. WIELEN \\ Astronomisches Rechen-Institut Heidelberg \\ AND \\ S. VON LINDEN \\ Landessternwarte Heidelberg-Königstuhl
}

We give a detailed description of the dynamical interaction of the stellar and gaseous components of a galactic disk. The stability of the two-component system against axisymmetric density perturbations is analyzed and the critical velocity dispersions of the stars and the gas, which control the instability, are determined. By comparison with the observed velocity dispersions it is shown that NGC 6946, a typical Sc galaxy, seems to be stable in the outer parts of the disk, but is dynamically unstable in the inner parts. The transition occurs exactly at the HII region disk boundary, so that the onset of gravitational instability appears to be related to the threshold of massive star formation (Kennicutt 1989). We study the dynamical evolution of the instability by numerical simulations using a code (cf. Casoli \& Combes 1982), which implements both the stellar and gaseous components. It is shown that the gas forms after a short initial period of growing ring-like perturbations large cloud complexes, which induce by 'swing-amplification' (Toomre 1990) multi-armed spiral structures in the stellar and gas disks, exactly as observed.

\section{Acknowledgements}

We thank A. Toomre for enlightening discusions and F. Combes for letting use us her code.

\section{References}

Casoli F. and Combes F. (1982) $A \& A$ 110, 287

Kennicutt R.C. (1989) ApJ 344, 685

Toomre A. (1990) in Dynamics and Interactions of Galaxies, R. Wielen (ed. ), Springer, p. 292 